EPJ manuscript No.

(will be inserted by the editor)

\title{
Recursion method and one-hole spectral function of the Majumdar-Ghosh model
}

\author{
R. O. Kuzian ${ }^{1}$, R. $\operatorname{Hayn}^{2,3}$, and J. Richter ${ }^{4}$ \\ 1 Institute for Materials Science, Ukrainian Academy of Sciences, Krzhizhanovskogo 3, 03180 Kiev, Ukraine \\ 2 Leibniz-Institut für Festkörper- und Werkstoffforschung Dresden, P.O.Box 270016, D-01171 Dresden, Germany \\ 3 Laboratoire Matériaux et Microélectronique de Provence, 49, rue Joliot-Curie, IRPHE, F-13384 Marseille Cedex 13, France \\ 4 Institut für Theoretische Physik, Otto-von-Guericke Universität Magdeburg, P.O.Box 4120, D-39016 Magdeburg, Germany
}

November 7, 2018

\begin{abstract}
We consider the application of the recursion method to the calculation of one-particle Green's functions for strongly correlated systems and propose a new way how to extract the information about the infinite system from the exact diagonalisation of small clusters. Comparing the results for several cluster sizes allows us to establish those Lanczos coefficients that are not affected by the finite size effects and provide the information about the Green's function of the macroscopic system. The analysis of this 'bulk-related' subset of coefficients supplemented by alternative analytic approaches allows to infer their asymptotic behaviour and to propose an approximate analytical form for the 'terminator' of the Green's function continued fraction expansion for the infinite system. As a result, the Green's function acquires the branch cut singularity corresponding to the incoherent part of the spectrum. The method is applied to the spectral function of one-hole in the Majumdar-Ghosh model (the one-dimensional $t-J-J^{\prime}$ model at $J^{\prime} / J=1 / 2$ ). For this model, the branch cut starts at finite energy $\omega_{0}$, but there is no upper bound of the spectrum, corresponding to a linear increase of the recursion coefficients. Further characteristics of the spectral function are band gaps in the middle of the band and bound states below $\omega_{0}$ or within the gaps. The band gaps arise due to the period doubling of the unit cell and show up as characteristic oscillations of the recursion coefficients on top of the linear increase.
\end{abstract}

PACS. PACS-75.10.Pq Spin chain models - PACS-71.10.Pm Fermions in reduced dimensions - PACS71.27.+a Strongly correlated electron systems; heavy fermions

\section{Introduction}

The one-hole spectral function (or the imaginary part of the one-particle Green's function) is a central quantity in the theory of strongly correlated systems since it contains the information about the charge dynamics in a correlated background [1. It was often studied in the theory of high-temperature superconductors (HTSC) using the several variants of the $t-J$ or the Hubbard model [2]. Other examples are the Kondo peak seen in the spectral function of heavy fermion compounds [4, or the features of spin-charge separation for one-dimensional problems [5]. The most important experimental method to measure the one-hole spectral function is angle-resolved photoemission.

A powerful method to calculate the one-hole spectral function for strongly correlated systems consists in the exact diagonalisation of small clusters [2] using the Lanczos method 3. That can be understood like a tridiagonalisation of the Hamilton matrix. The method and its application in electronic structure theory has already a long history. It is also known as recursion method according to pioneering studies of Haydock and coworkers in the sev- enties 6]7. That time, however, it was mostly applied to different problems of noninteracting electrons. The numerous spectral function calculations using the different versions of the $t-J$ or the Hubbard model [2] have usually very large finite size effects. Normally, they provide the spectral function only as a sequence of well separated $\delta$-peaks. On the other hand, it is already known for long time that the recursion method has the potential to provide the information about the bulk spectral function by a proper termination of the continued fraction representation of the Green's function [8]. That idea was used in the field of many-body dynamics for pure spin systems by Viswanath and Müller [10].

Below we would like to present an improvement of the recursion method for the charge dynamics in strongly correlated systems. By finding the correct asymptotic behaviour of the recursion coefficients $a_{n}$ and $b_{n}$ for $n \rightarrow$ $\infty$ which is not influenced by boundary effects we are able to extract the bulk spectral function. The method will be demonstrated for the example of one-hole in the Majumdar-Ghosh (MG) model, i.e. in the 1D spin chain with exchange terms between first $J$ and second neigh- 
R. O. Kuzian et al.: Recursion method and one-hole spectral function of the Majumdar-Ghosh model

bours $J^{\prime}$ at the special ratio $J^{\prime} / J=1 / 2$. It has the advantage that one knows explicitly the ground-state wave function of the spin system 11. The MG model is considered here as a generic model for a 1D spin chain with a spin gap. That means that the spin correlation functions decay exponentially. Therefore, the MG model is a good candidate to present our method since the fast decay of spin correlation functions tends to minimise the boundary effects on the recursion coefficients. We analyse also the recursion coefficients corresponding to a recently presented variational study of the one-hole spectral function using the subspace of one-spinon wave-functions [12. It completes our approach by providing additional information on the asymptotic behaviour of recursion coefficients and on the spectral function. The main physical question connected with the MG model is: how are the features of spin-charge separation in 1D modified by a spin gap. We would like to present a rather complete description of the one-hole spectral function of the MG model. The paper is organised as follows: first we recall the recursion method (Sec. 2) and introduce the one-dimensional $t-J-J^{\prime}$ model (Sec. [3). In Sec. 4 we collect analytical and numerical results in the strong coupling limit $J, J^{\prime} \rightarrow 0$ and in Sec. $\mathrm{V}$ we present the asymptotic behaviour of the recursion coefficients. That analysis is used to reconstruct the spectral function (Sec. 6) and to calculate the bound states and band gaps (Sec. (7).

\section{The Recursion Method}

The recursion method (RM) 6 7 is a powerful tool to calculate the matrix elements of the resolvent $(z-\hat{H})^{-1}$ of a Hamiltonian $\hat{H}$. Given an arbitrary starting state $\left|u_{0}\right\rangle$, the RM generates a new basis in which the Hamiltonian matrix is tridiagonal. One can formulate the RM in two ways, in the Hamiltonian or the Liouvillian representation [10. The latter one corresponds to a generalisation of the $\mathrm{RM}$ to the Liouvillian space of operators. Usually, it is used for finite temperature problems. In the present case, however, both formulations will be demonstrated to be identical and we concentrate on the Hamiltonian variant.

In the Hamiltonian representation, the RM starts from a vector in Hilbert space, i.e. from some wave function of the system $\left|u_{0}\right\rangle$. The new basis (orthogonal, but not normalised) is generated according to the Lanczos procedure

$$
\left|u_{n+1}\right\rangle=\left(\hat{H}-a_{n}\right)\left|u_{n}\right\rangle-b_{n}^{2}\left|u_{n-1}\right\rangle
$$

with $\left|u_{-1}\right\rangle=0$, and $b_{0}^{2}=\left\langle u_{0} \mid u_{0}\right\rangle$. The coefficients are calculated from

$$
a_{n}=\left\langle u_{n}|\hat{H}| u_{n}\right\rangle /\left\langle u_{n} \mid u_{n}\right\rangle, b_{n}^{2}=\left\langle u_{n+1} \mid u_{n+1}\right\rangle /\left\langle u_{n} \mid u_{n}\right\rangle
$$

Having calculated the recursion coefficients $a_{n}$ and $b_{n}$, one can easily find the matrix element of the resolvent with $\left|u_{0}\right\rangle$ :

$$
R(z)=\left\langle u_{0}\left|(z-\hat{H})^{-1}\right| u_{0}\right\rangle
$$

$$
=\frac{b_{0}^{2}}{z-a_{0}-\frac{b_{1}^{2}}{z-a_{1}-\frac{b_{2}^{2}}{\cdot}}} \equiv \frac{b_{0}^{2}}{z-a_{0}-} \frac{b_{1}^{2}}{z-a_{1}-} \cdots .
$$

For a starting state corresponding to one hole in the correlated state, the resolvent $R(z)$ is identical to the oneparticle Green's function and its imaginary part gives the spectral function we are interested in.

In this form the RM was applied to many body problems (see e.g. Refs. 13, 2,14 and references therein) several times. In these cases the resolvent matrix elements of the full many body Hamiltonian were calculated. This work can be done numerically for finite systems. Unfortunately, at present, such calculations are only possible for very small clusters. The resulting $R(z)$ may be related with the dynamic susceptibility [13. The problem is that $R(z)$ for a finite system is a rational function that may be represented as a set of poles. The corresponding spectral density consists of a set of $\delta$-functions which are usually represented using an artificial broadening. This is quite different from that what we can expect for a macroscopic system of interacting particles. The spectrum of a nontrivial interacting system contains usually a continuous part (the "continuum" which is connected with a branch cut in $R(z)$ ) and eventually one or several isolated states (the point spectrum)). A sequence of broadened $\delta$-functions is a very poor approximation for the continuous part of the spectrum. Furthermore, such a procedure neglects completely the important difference between the continuum and isolated poles. All the fine structure of the spectrum is completely lost.

Below we will show what kind of information about the macroscopic system may be extracted from the exact diagonalisation studies of small clusters and propose a more physical procedure of smoothing the spectral density. The ED has been performed for the $1 \mathrm{D} t-J-J^{\prime}$ model with $12,16,20$, and 24 lattice sites according to the method described in Refs. 13.2. From these calculations we extract the information about the bulk related asymptotic behaviour of the recursion coefficients $a_{n}$ and $b_{n}$ for large $n$. Additional information about the asymptotics can be obtained using the variational approach presented in Ref. [12].

\section{Majumdar-Ghosh model}

In order to demonstrate our method, we specialise to the calculation of the one-hole spectral function in the onedimensional Majumdar-Ghosh model (the 1D $t-J-J^{\prime}$ model with $J^{\prime} / J=1 / 2$ ). The interest to $1 \mathrm{D}$ models has been revived after the creation of quasi one dimensional transition metal compounds. The reports on angle-resolved photoemission spectroscopy (ARPES) 15 studies show that the most striking theoretical prediction for 1D strongly correlated systems, the spin-charge separation, can be observed. The interest to this phenomenon has increased after Anderson's proposal that it explains the properties of 
2D cuprate superconductors [16. The calculation of oneparticle Green's functions for 1D strongly correlated system is a complex and non-trivial task. The complete answer is absent even for the exactly solvable 1D Hubbard model 17. That is why the development of simple approximate approaches remains important. In the present study we consider the $t-J-J^{\prime}$ model, which describes the basic physics of the electron motion in $1 \mathrm{D}$ transition metal compounds

$$
\hat{H}=\hat{t}+\hat{J}+\hat{J}^{\prime},
$$

where

$$
\begin{gathered}
\hat{t}=-t \sum_{i, \alpha}\left(X_{i}^{\alpha 0} X_{i+1}^{0 \alpha}+h . c .\right) \\
\hat{J}=\frac{J}{2} \sum_{i, \alpha, \beta} X_{i}^{\alpha \beta} X_{i+1}^{\beta \alpha}, \hat{J}^{\prime}=\frac{J^{\prime}}{2} \sum_{i, \alpha, \beta} X_{i}^{\alpha \beta} X_{i+2}^{\beta \alpha},
\end{gathered}
$$

and we introduce Hubbard projection operators that act in the subspace of on-site states, namely boson-like operators

$$
X_{i}^{\alpha \beta} \equiv|\alpha, i\rangle\langle\beta, i|, \alpha, \beta=\uparrow, \downarrow,
$$

and fermion-like ones $X_{i}^{\alpha 0}, i$ being the site index. To make contact with the standard notation we note that

$$
\frac{1}{2} \sum_{\alpha, \beta} X_{i}^{\alpha \beta} X_{j}^{\beta \alpha}=\mathbf{S}_{i} \mathbf{S}_{j}+\frac{1}{4} n_{i} n_{j},
$$

for $i \neq j$, where $\mathbf{S}_{i}$ and $n_{i}$ are spin and density operators, respectively. The exchange term to second neighbours $J^{\prime}$ is especially relevant in $1 \mathrm{D}$ compounds with a spin-Peierls transition, like $\mathrm{CuGeO}_{3}$. For $J^{\prime} / J$ larger or equal 0.2411 the quasi long-range order (algebraically decaying spincorrelation functions) of the 1D antiferromagnetic spinhalf Heisenberg model is destroyed and one observes a gap in the spin-excitation spectrum 18 .

Our aim is to calculate the one-particle two-time retarded Green's function

$$
G(k, z)=\left\langle\left\langle X_{k}^{\sigma 0} \mid X_{k}^{0 \sigma}\right\rangle\right\rangle
$$

for $z=\omega+\imath 0^{+}$and the spectral density

$$
A(k, \omega)=-\frac{1}{\pi} \operatorname{Im} G\left(k, \omega+\imath 0^{+}\right),
$$

where $X_{k}^{\sigma 0}=\sqrt{2 / L} \sum_{m} \mathrm{e}^{-\imath k m} X_{m}^{\sigma 0}$, and $L$ is the number of sites, the factor $\sqrt{2}$ is introduced for the purpose of normalisation. The above one-particle Green's function describes the motion of one hole in the correlated state described by the Majumdar-Ghosh wave function [1] (from now on we consider the special case $J^{\prime} / J=1 / 2$ ). The notation means

$$
\langle\langle A \mid B\rangle\rangle \equiv-i \int_{t^{\prime}}^{\infty} d t \mathrm{e}^{i \omega\left(t-t^{\prime}\right)}\left\langle\left\{A(t), B\left(t^{\prime}\right)\right\}\right\rangle,
$$

with $\{\ldots$,$\} denoting the anticommutator, and where the$ expectation value means the thermal average over a grand canonical ensemble: $\langle\ldots\rangle=Q^{-1} S p\left[\mathrm{e}^{-\beta(H-\mu N)} \ldots\right], \quad Q=$
$S p \mathrm{e}^{-\beta(H-\mu N)}$. Here $S p$ implies the trace of an operator, $N$ is the particle number operator, $\beta=(k T)^{-1}$ is an inverse temperature, and $\mu$ represents the chemical potential. The time dependence of the operator $A(t)$ is given by $A(t)=\mathrm{e}^{i t(H-\mu N)} A \mathrm{e}^{-i t(H-\mu N)}$. At zero temperature, $\langle\ldots\rangle$ goes over into the expectation value with the groundstate wave function $\left|\Psi_{0}\right\rangle$. It is not difficult to see that in the given case the one-particle Green's function (5) may be formulated as a resolvent matrix element

$$
G(k, z)=\left\langle X_{k}^{\sigma 0}\left|\left(z+E_{0}-\hat{H}\right)^{-1}\right| X_{k}^{\sigma 0}\right\rangle,
$$

with the state $\left|X_{k}^{\sigma 0}\right\rangle=X_{k}^{\sigma 0}\left|\Psi_{0}\right\rangle$ denoted in the same way as the corresponding operator. It means also that the Hamiltonian and Liouvillian formulation of the RM are now equivalent if we take into account the shift of the diagonal recursion coefficients $a_{n}$ by the ground-state energy $E_{0}$ of the pure spin system.

When the ground state is degenerate, as it is the case in the Majumdar-Ghosh model, we have to take $S p$ over the ground-state manifold in the calculation of $\langle\ldots\rangle$ in (17). We have found that the spectral function calculated for two orthogonal ground states of the model according to the prescription given in Sec. [6 coincides within the accuracy of the method.

\section{Strong coupling limit}

For the following analysis it is important to recall some former results for the one-hole spectral function in the MG model using a variational ansatz [12. It was shown that one can give an exact result in the strong coupling limit $J, J^{\prime} \rightarrow 0$. In this limit only the $\hat{t}$ operator remains in Eq. (4). Note that it is a true many-body Hamiltonian due to the constraint of no double occupancy, it is often called the $\hat{t}$-model. Formally, the $\hat{t}$-model is the limiting case of various models $(U \rightarrow \infty$ of the Hubbard model, $J \rightarrow 0$ of the $t-J$ model, etc.), and its ground state is strongly degenerate. The degeneration is removed by an infinitesimal perturbation that fixes the ground state. It is clear that different spin-models give different answers for the Green's function (5) and the spectral density (6) in the limit of the $\hat{t}$-model since they may differ by the spin ground state 12 .

The exact result was found using the basis operator set

$$
v_{m, r}=\sqrt{\frac{2}{L}} \sum_{\alpha_{1}, \ldots, \alpha_{r}} X_{m}^{\sigma \alpha_{1}} X_{m+g}^{\alpha_{1} \alpha_{2}} \ldots X_{m+r-g}^{\alpha_{r-1} \alpha_{r}} X_{m+r}^{\alpha_{r} 0},
$$

$g=r /|r|$. The operator $v_{m, r}$ may be interpreted as holon ('right end' $\ldots X_{m+r}^{\alpha_{r} 0}$ ) and spinon ('left end' $X_{m}^{\sigma \alpha_{1}} \ldots$ ) excitations connected by a string of spin 'flips' (in fact, this string contains also diagonal spin operators but they also create excitations in a quantum antiferromagnet). The double Fourier transform over the $m$ and $r$ indices gives exact eigenstates of the $\hat{t}$-term for any magnetic background. In the case of finite exchange values, the basis set 
(9) is not complete and corresponds to the single spinon approximation.

It was shown that the Green's function (5) for fixed $k$ has a formal analogy with the on-site Green's function of the 1D tight-binding model of non-interacting electrons in a non-orthogonal basis

$$
\begin{aligned}
& G(k, z)=\frac{1}{L} \sum_{Q} \frac{2 Z(Q)}{z+\epsilon_{h}(k-Q)} \\
& =\frac{1}{\sqrt{z^{2}-4 t^{2}}}\left[1+\sum_{n=1}^{\infty} 2 \cos k n\left\langle\Omega_{0 \rightarrow n}\right\rangle \tau^{n}(z)\right],
\end{aligned}
$$

where $\epsilon_{h}(k) \equiv 2 t \cos (k)$.

The spectral function may be represented like

$$
A(k, \omega)=\int_{-\pi}^{\pi} \frac{d Q}{\pi} Z(Q) \delta\left(\omega+\epsilon_{h}(k-Q)\right),
$$

with

$$
\begin{aligned}
& Z(Q) \equiv \frac{1}{2} \sum_{n=-\infty}^{\infty} \exp [-\imath(Q-\pi) n]\left\langle\Omega_{0 \rightarrow n}\right\rangle \\
& \Omega_{0 \rightarrow r}=\sum_{\alpha_{1}, \ldots, \alpha_{r}, \sigma} X_{0}^{\sigma \alpha_{1}} \ldots X_{r-g}^{\alpha_{r-1} \alpha_{r}} X_{r}^{\alpha_{r} \sigma} \\
& =\left(2 \mathbf{S}_{0} \mathbf{S}_{g}+\frac{1}{2}\right)\left(2 \mathbf{S}_{g} \mathbf{S}_{2 g}+\frac{1}{2}\right) \ldots\left(2 \mathbf{S}_{r-g} \mathbf{S}_{r}+\frac{1}{2}\right) \\
& \tau(z) \equiv \frac{z-\sqrt{z^{2}-4 t^{2}}}{2 t}=\frac{t}{z-} \frac{t^{2}}{z-} \frac{t^{2}}{z-} \cdots .
\end{aligned}
$$

Integrating over the $\delta$-function, the spectral function (11) may be given in the form [19]

$$
A(k, \omega)=\frac{Z(k+\phi)+Z(k-\phi)}{\pi \sqrt{4 t^{2}-\omega^{2}}}, \quad \cos (\phi)=-\frac{\omega}{2 t} .
$$

The expressions (1113) imply that the spectrum is continuous and bounded on the interval $-2 t \leq \omega \leq 2 t$ for all the possible spin models in the considered limit. It may be interpreted as holon motion with the dispersion $\epsilon_{h}(k)$ with an immovable spinon. The difference in hole spectral densities and Green's functions arises due to the expectation value $Z(Q)$ that depends on the spin part of the Hamiltonian. Some parts of the spectrum may be excluded by a vanishing $Z(Q)$.

For the 1D pure (non frustrated) antiferromagnetic Heisenberg model, $Z(Q)$ was given in Ref. [20,19] as $Z(Q)$ $\propto \Theta(Q-\pi / 2) / \sqrt{Q-\pi / 2}$. The square-root singularity leads to additional peaks in the spectral function, such that for each $k$ value one can distinguish well pronounced spinon and holon peaks (for details see also Ref. 21]). For the Majumdar-Ghosh (MG) wave function, on the other hand, one finds $Z(Q)=\frac{3}{2}(1+\cos Q) /(5+4 \cos 2 Q)$ (see Ref. 22] ) where $Z(Q)$ is non zero for all values of $Q$. The peak at $Q=\pi / 2$ becomes very wide. That leads to a large damping of the holon peak [12]. One finds the following explicit formula for the spectral function of the MG model in the limit $J, J^{\prime} \rightarrow 0$ :

$$
\begin{aligned}
& A(k, \omega)=\frac{3}{8 t \pi \sqrt{1-x^{2}}} \cdot \\
& \frac{20+4 x \cos k+16\left(2 x^{2}-1\right) \cos 2 k-8 D}{25+40\left(2 x^{2}-1\right) \cos 2 k+8 \cos 4 k+8\left(8 x^{4}-8 x^{2}+1\right)} \\
& x \equiv \frac{\omega}{2 t}, \quad D \equiv x \cos 3 k+4 x^{3} \cos k
\end{aligned}
$$

which is non zero for all $-2 t \leq \omega \leq 2 t$.

Now, for the MG model, we compare the continued fraction expansion of the one-particle Green's function for the macroscopic system (10) with that for a finite ring. The summation of series in (10) gives

$$
G(k, z)=\frac{4-2\left[2 \tau(z)+\tau^{3}(z)\right] \cos k-\tau^{4}(z)}{\sqrt{z^{2}-4 t^{2}}\left[4+4 \cos 2 k \tau^{2}(z)+\tau^{4}(z)\right]},
$$

that may be rewritten using the identity $\tau(z)=t /(z-$ $t \tau(z))$ in the form

$$
G(k, z)=\frac{1}{z-a_{0}-} \frac{b_{1}^{2}}{z-a_{1}-b_{2}^{2} t^{-1} \tau(z)[1+F(z)]},
$$

where

$$
\begin{aligned}
& a_{0}=\frac{t}{2} \epsilon, b_{1}^{2}=3 t^{2}\left(1-\frac{3}{4} \epsilon^{2}\right), a_{1}=\frac{9 \epsilon^{3}-6 \epsilon}{8 b_{1}^{2}} t^{3}, \\
& b_{2}^{2}=\frac{9\left(8-7 \epsilon^{2}\right) t^{4}}{16 b_{1}^{2}}, \epsilon \equiv \cos k,
\end{aligned}
$$

and the function $F(z)$ :

$$
\begin{aligned}
F(z)= & \frac{\epsilon\left(1-\epsilon^{2}\right) V(z)}{\left(8-7 \epsilon^{2}\right) W(z)}, \\
& V(z) \equiv z\left(18 \epsilon^{4}-27 \epsilon^{2}+8\right)+4 \epsilon\left(10-9 \epsilon^{2}\right) \\
& -3\left(12 \epsilon^{4}-21 \epsilon^{2}-24\right) \tau(z) \\
& W(z) \equiv 2 z^{2}\left(4-3 \epsilon^{2}\right)-2 z \epsilon-12 \\
& +11 \epsilon^{2}-\left[2 z\left(4-3 \epsilon^{2}\right)-\epsilon\right] \tau(z),
\end{aligned}
$$

vanishes for $k=0, \pm \frac{\pi}{2}, \pm \pi$. For these $k$ values we thus readily obtain $G(k, z)$ in the continued fraction form (3) with $a_{n}=a_{\infty}=0, b_{n}^{2}=b_{\infty}^{2}=t^{2}, n>2$ that follows from (13). For other $k$ values the coefficients asymptotically tend to the same constant limits $a_{\infty}, b_{\infty}$.

Fig. 1 compares the analytical result for $a_{n}, b_{n}$ with the values provided by the ED of a ring with $L=24$ sites at $J=0.01$. One sees that the values of $a_{n}$ with $n \leq 10$ are not influenced by the boundary. These first values give us bulk-related information. Comparing with other cluster sizes $L$, one finds that $a_{n}$ has no finite size effect for $n \leq(L-4) / 2$. For the off-diagonal elements $b_{n}$ one has $n \leq(L-4) / 2+1$. So, we may conclude that ED studies of finite systems are able to provide the information that concerns the macroscopic system and may be used for the analysis of the spectral density. Despite the small number of 'bulk related' coefficients they definitely reached their asymptotic regime and may be extrapolated to $n \rightarrow \infty$ 


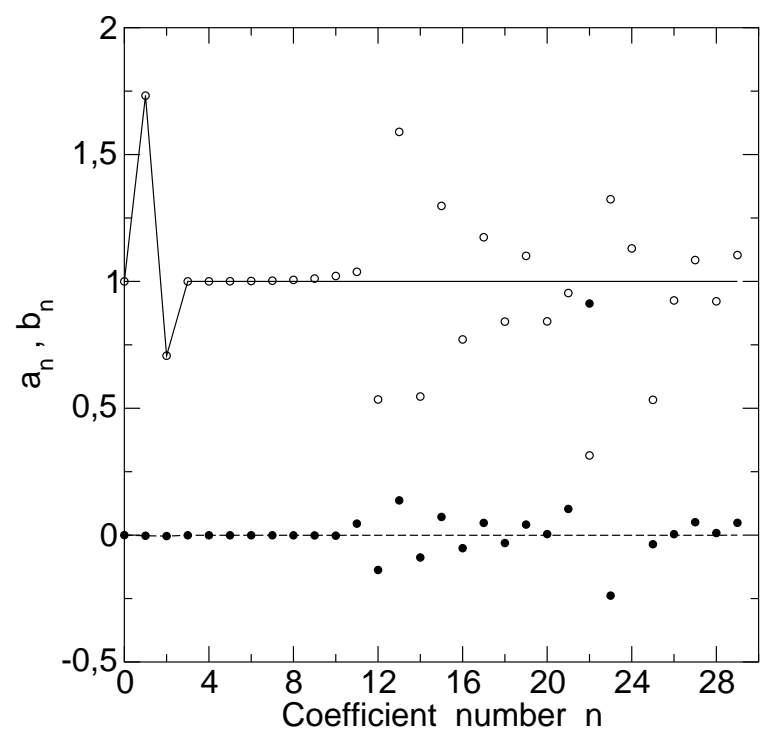

Fig. 1. The coefficients $a_{n}(k)$ (filled circles) and $b_{n}(k)$ (open circles) of the continued fraction expansion of the one-particle Green's function $G(k, \omega)$ versus $n$ for $k=\pi / 2, J=0.01$ provided by the exact diagonalisation of the $L=24$ sites $t-J-J^{\prime}$ ring compared with the result for the single spinon approximation (solid or dashed line, exact for $J=0, L=\infty$ ). The hopping parameter $t=1$ is the unit of energy.

giving the exact Green's function and spectral density for infinite chain. The situation is not always so simple, but this example clearly shows a manageable way to extrapolate ED results to the macroscopic system.

Concluding the consideration of the strong coupling limit we recall that it is commonly believed that the main changes in the spectral density at larger finite couplings (i.e. $J \neq 0$ ) come from the appearance of the spinon dispersion $\epsilon_{s}$, and may be accounted for by the substitution

$$
\epsilon_{h}(k-Q) \rightarrow \epsilon_{h}(k-Q)+\epsilon_{s}(Q)
$$

in Eqs. (1011) 19 21]. Below we shall demonstrate that holon and spinon scattering lead to additional changes: the continuous part of the spectrum expands up to infinite energies and for the case of the MG model, additional discrete states appear.

\section{Termination of the continued fraction}

Now we concentrate on results for finite $J, J^{\prime}$. In the previous section we have shown that the few first recursion coefficients given by the exact diagonalisation study of a small cluster are (almost) not affected by boundaries. Thus, in order to infer the shape of the spectral density for macroscopic system we should rely on these 'bulk related' coefficients. To define the bulk related coefficients we observe from Fig. 2a that the coefficients for $L=12$ start to deviate from the common line at $n=4$, those for $L=16$ at $n=6$ and for $L=20$ at $n=8$. The corresponding numbers for $b_{n}$ (Fig. 2b) are 5, 7, and 9. Generalising we find $a_{n}: n<(L-4) / 2$ and $b_{n}: n<(L-4) / 2+1$ to be bulk related coefficients. From the inset of Fig. 2a we clearly see that 'bulk related' coefficients for finite $J, J^{\prime}$ show a different tendency (to grow with moderate oscillations) compared to the rest of the sequence for a cluster (strong oscillations around a constant value).

For finite $J$ the number of bulk related coefficients is slightly reduced in comparison to Fig. 1. It should be noted that the 'bulk related' coefficients with large $n$ have still a small finite size effect, but that is several orders of magnitude smaller than for the rest of the sequence. We have also observed that in the case of the pure $t-J$ model (i.e. $\left.J^{\prime}=0\right)$ the situation is worse. The number of recursion coefficients that are not influenced by finite size effects is considerably reduced. One may recall that according to Eq. (2) the coefficients are related to static spin correlation functions of the spin background and speculate that the difference arises due to the algebraically decaying spin correlation functions for the 1D Heisenberg model in difference to the exponential decay for the MG model.

For the calculation of the spectral density we will try to extrapolate the tendency shown by the 'bulk related' coefficients. In other words, we rewrite the expression (3) in the form

$$
G(k, z)=\frac{b_{0}^{2}}{z-a_{0}-} \frac{b_{1}^{2}}{z-a_{1}-} \cdots \frac{b_{n_{0}}^{2}}{z-a_{n_{0}}-T_{n_{0}}(k, z)},
$$

and try to find a function $\tilde{T}_{n_{0}}$ (a so called "terminator") that is close to $T_{n_{0}}$. The terminator should have such an analytic behaviour which corresponds to the asymptotics of $a_{n}$ and $b_{n}$.

Various ways to construct such approximations are described in the literature on the recursion method (see Refs. [8, $9,23,24]$ ). The asymptotic behaviour of continued fraction coefficients is governed by the band structure and the singularities of the spectral density 24]. The problem is well studied for a bounded spectrum: $\left\{a_{n}\right\}$ and $\left\{b_{n}\right\}$ converge toward limits in the single band case, oscillate endlessly in a predictable way in the multiband case. Damped oscillations are created by isolated singularities. But the growth of coefficients indicates that the spectrum we deal with is unbounded. The infinite growth of diagonal coefficients is observed for the $t-J_{z}$ model that differs from the $t-J$ model by omitting spin fluctuations, $a_{n}=n J_{z}, b_{n}^{2}=z_{0} t^{2},\left(z_{0}\right.$ is the number of nearest neighbours) [25]. This growth has a clear physical meaning: a hole moving on the Neel background (the ground state for $t-J_{z}$ model without holes) creates a string of overturned spins; every $\left|u_{n}\right\rangle$ state (11) is the combination of such strings containing $n$ overturned spins, thus its en- 

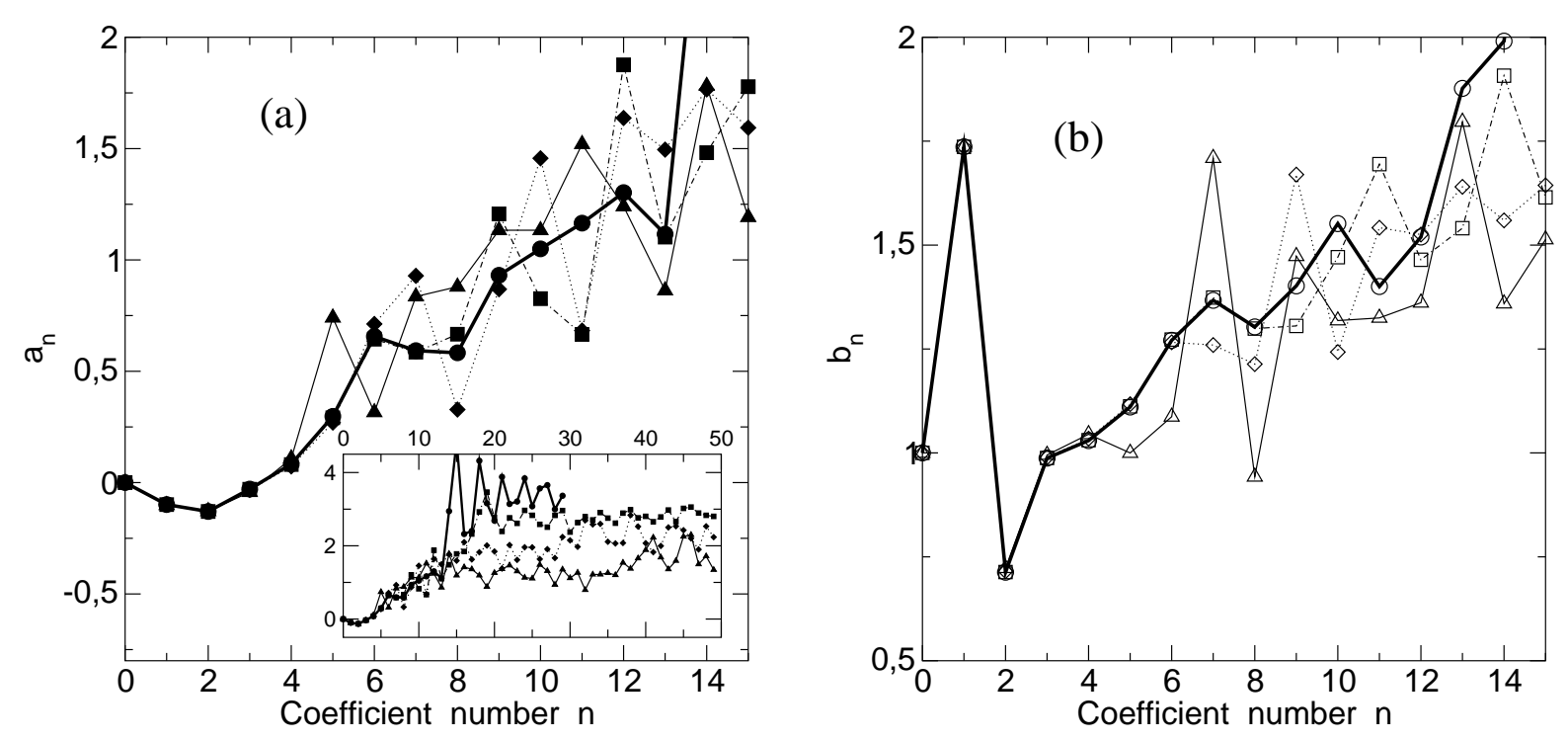

Fig. 2. The coefficients $a_{n}(k)$ (full symbols, (a)), and $b_{n}(k)$ (open symbols, (b)) of the continued fraction expansion of the one-particle Green's function $G(k, \omega)$ versus $n$ for $k=\pi / 2, J=0.4$ and various lattice sizes $L=12$ (triangles and thin solid line), 16 (diamonds and dotted line), 20 (squares and dash-dotted line), 24 (circles and thick solid line). The insert in panel (a) shows $a_{n}(k)$ on a larger scale.

$\operatorname{ergy}\left\langle u_{n}\left|\hat{t}+\hat{J}_{z}\right| u_{n}\right\rangle \propto n J_{z}$. The non-diagonal coefficients $b_{n}$ are constant. As a result, the hole is localised in the $t-J_{z}$ model, its spectral function does not depend on quasimomentum $k$ and consists of an infinite set of $\delta$ functions. The spectrum is discrete and unbounded [27].

When spin fluctuations are added the situation becomes more complicated, now the non-diagonal coefficients also grow. Such a situation was studied in the two-dimensional $t-J$ model as well as for spin-fermion models of the $\mathrm{CuO}_{2}$ plane, where the following asymptotics was found within the framework of the self-consistent Born approximation [29,30]:

$b_{n} \approx \lambda_{1} n+\lambda_{2}, \quad a_{n} \approx 2 \lambda_{1} n+\lambda_{3}, \quad \lambda_{i}=\lambda_{i}(k), \quad n \gg 1$.

It indicates the existence of an exponential tail in the spectral density. But the connection between an unbounded spectrum (toward high energies) and the growth of recursion coefficients is far more general. For example, the model spectral density

$$
A(\omega)=c^{t} \omega^{\rho} \exp \left(-\omega^{\beta}\right), c(\beta)=\frac{2 \Gamma(\beta)}{[\Gamma(\beta / 2)]^{2}}, \omega>0,
$$

with the parameters $t, \rho$, and $\beta$, gives the following asymptotic behaviour of the recursion coefficients 24]:

$$
b_{n} \approx\left(\frac{n}{c(2 \beta)}\right)^{1 / \beta}, \quad a_{n} \approx 2\left(\frac{n}{c(2 \beta)}\right)^{1 / \beta},
$$

which does not depend on $t$ and $\rho$. For $\beta=1$ we have a linear dependence on $n$ for $\left\{a_{n}\right\}$ and $\left\{b_{n}\right\}$ [7].

The linear growth holds also for the MG model. Fig. 3 shows the linear contributions to the growth of 'bulk related' coefficients. We have found $\lambda_{1}, \lambda_{2}$ using least mean square fit of the $\left\{b_{n}\right\}$ sequence and only $\lambda_{3}$ was adjusted for $\left\{a_{n}\right\}$. Fig. 3 shows that our assumption about the slope $d a_{n} / d n \approx 2 d b_{n} / d n$ holds.

The continued fraction expansion of the incomplete Gamma function has the same asymptotics (20) and is written as 31

$$
\begin{aligned}
& \Gamma(\alpha, x)=\frac{\mathrm{e}^{-x} x^{\alpha}}{x+1-\alpha-} \frac{1 \cdot(1-\alpha)}{x+3-\alpha-} \frac{2 \cdot(2-\alpha)}{x+5-\alpha-} \cdots \\
& \cdots \frac{n \cdot(n-\alpha)}{x+(2 n+1-\alpha)-\cdots} .
\end{aligned}
$$

We shall use this circumstance for the construction of the terminator $\tilde{T}_{N}(k, z)$ for $G(k, z)$ (19). 
R. O. Kuzian et al.: Recursion method and one-hole spectral function of the Majumdar-Ghosh model
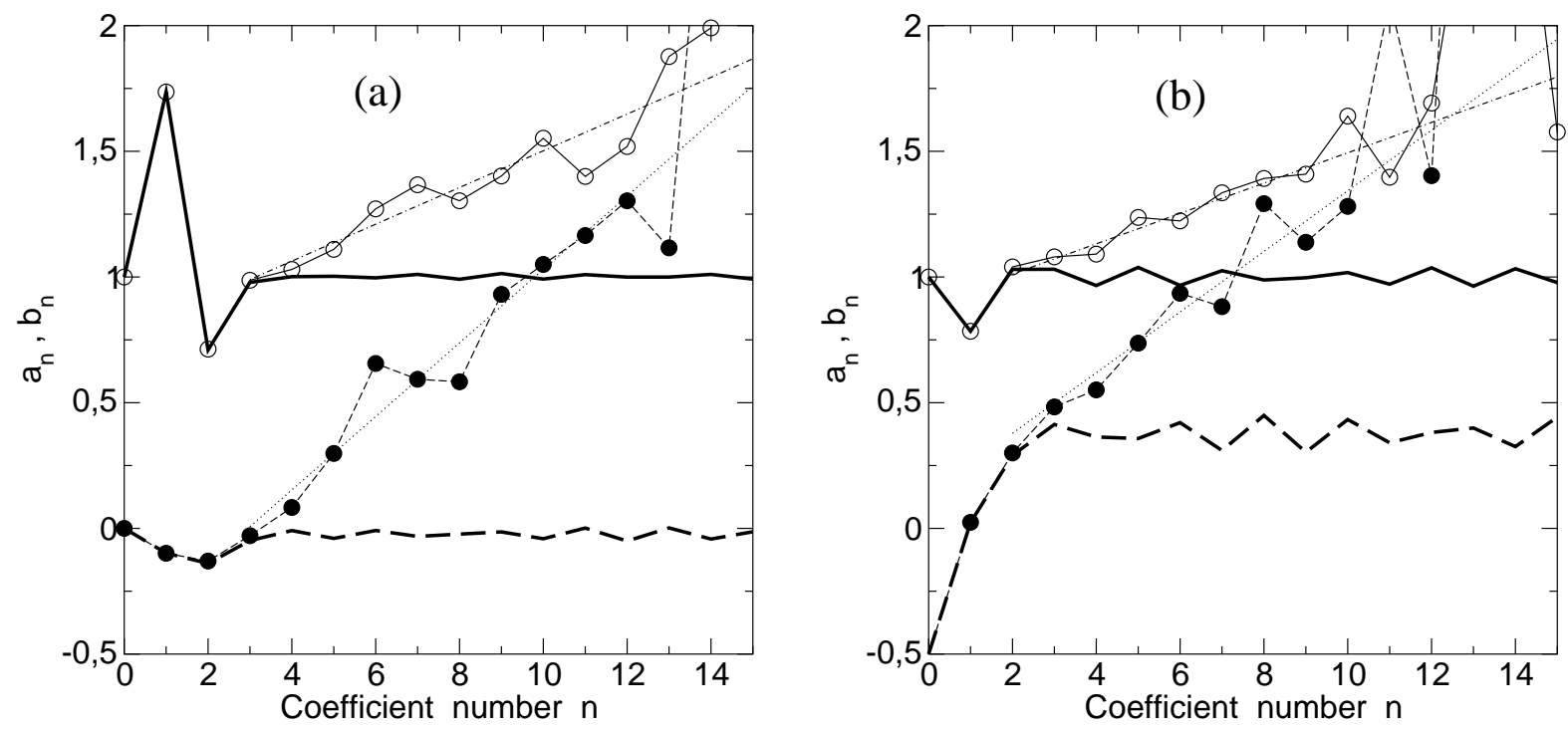

Fig. 3. Comparison of the coefficients $a_{n}(k)$ (filled circles joined by dashed lines) and $b_{n}(k)$ (open circles, solid lines) provided by the exact diagonalisation of the $L=24$ sites $t-J-J^{\prime}$ ring with the result of the single spinon approximation (thick dashed and solid lines, correspondingly) for finite $J=0.4$. The quasimomenta are $k=\pi / 2$ (a) and $k=0$ (b). The linear contributions in the asymptotics of the exact coefficients $\left(n \rightarrow \infty: \tilde{b}_{n}=c_{1} n+c_{2}, \tilde{a}_{n}=2 c_{1} n+c_{3}\right)$ are shown by dashed-dotted and dotted lines, correspondingly.

Let us introduce the model Green's function

$$
\tilde{G}(k, z)=-\frac{\Gamma\left(\alpha, x \mathrm{e}^{-\imath \pi}\right) \mathrm{e}^{\imath \pi \alpha}}{\lambda_{1} \mathrm{e}^{x} x^{\alpha}},
$$

with the spectral density

$$
\tilde{A}(k, \omega)=\frac{1}{\lambda_{1} \Gamma(1-\alpha)}\left[\frac{\left(\omega-\omega_{0}\right)}{\lambda_{1}}\right]^{-\alpha} \exp \left[-\frac{\left(\omega-\omega_{0}\right)}{\lambda_{1}}\right] \text {. }
$$

Here $x=\left(z-\omega_{0}\right) / \lambda_{1}$,

$$
\omega_{0} \equiv \lambda_{3}-2 \lambda_{2}-\lambda_{1}
$$

is the lower bound of the continuous spectrum, $\alpha=2(M-$ $\left.\lambda_{2} / \lambda_{1}\right), M$ is the integer part of $\lambda_{2} / \lambda_{1}$. The continued fraction expansion coefficients for $\tilde{G}(k, z)$ are

$$
\begin{aligned}
& \tilde{a}_{m}=\lambda_{1}(2 m+1-\alpha)+\omega_{0}, m \geq 0, \tilde{b}_{0}=1, \\
& \tilde{b}_{m}=\lambda_{1} \sqrt{m(m-\alpha)} \approx \lambda_{1}(m-\alpha / 2), m>0 .
\end{aligned}
$$

Comparing with the definition (20) we find

$$
b_{n} \approx \tilde{b}_{M+n}, a_{n} \approx \tilde{a}_{M+n}
$$

Then we slightly generalise the recipe of Ref. 8] and approximate 30

$$
\begin{aligned}
\tilde{T}_{M+n_{0}}(k, z) & =\frac{\tilde{q}_{M+n_{0}-1}(k, z)-\tilde{G}(k, z) \tilde{p}_{M+n_{0}}(k, z)}{\tilde{q}_{M+n_{0}-2}(k, z)-\tilde{G}(k, z) \tilde{p}_{M+n_{0}-1}(k, z)} \\
& \approx T_{n_{0}}(k, z) .
\end{aligned}
$$

Here $\tilde{p}_{n}(k, z), \tilde{q}_{n}(k, z)$ are polynomials of first and second kind orthogonal with respect to the spectral density (23). They are calculated by the recursion relation 7,32

$$
\begin{aligned}
& \tilde{p}_{-1}=\tilde{q}_{-1}=0, \quad \tilde{p}_{0}=1, \quad \tilde{q}_{0}=\tilde{b}_{0}^{2}, \\
& \tilde{p}_{n}=\left(z-\tilde{a}_{n-1}\right) \tilde{p}_{n-1}-\tilde{b}_{n-1}^{2} \tilde{p}_{n-2}, \\
& \tilde{q}_{n-1}=\left(z-\tilde{a}_{n-1}\right) \tilde{q}_{n-2}-\tilde{b}_{n-1}^{2} \tilde{q}_{n-3} .
\end{aligned}
$$

$\tilde{p}_{n}(k, z)$ are proportional to the generalised Laguerre polynomials. In our calculations it holds always: $\lambda_{2} / \lambda_{1}>0$ and $-1<\alpha<0$.

We thus obtain $G(k, z)$ in the whole complex energy plane. It has the correct analytic properties and coincides with the retarded Green's function for $z$ in the upper half plane and with the advanced Green's function for $z$ in the lower half plane. It has a branch cut on the real axis for 
$\omega_{0}<z<\infty$ that corresponds to the continuum part of the spectrum. In addition, it may have isolated poles for $z<\omega_{0}$ which represent quasiparticle excitations.

Figs. 2 and 3 show that the recursion coefficients oscillate around their asymptotics. The influence of these oscillations on the spectral density was neglected so far. For a bounded spectrum this is the indication of a multiband spectrum. The problem was well studied in Refs. 9, 24. In the case of one gap, i.e. a spectrum lying in the disjoint intervals $E_{1} \leq \omega \leq E_{2}$ and $E_{3} \leq \omega \leq E_{4}\left(E_{2}<E_{3}\right)$ any pair of neighbouring coefficients $(y, x)=\left(b_{n}^{2}, a_{n}\right)$ or $\left(b_{n+1}^{2}, a_{n}\right)$ obeys the law 9 ]

$$
\left(x^{2}+A_{1} x+A_{2}+2 y\right)^{2}=X\left(-A_{1}-x\right),
$$

with

$$
\begin{aligned}
& A_{1} \equiv \frac{1}{2} \sum_{i} E_{i}, A_{2} \equiv \frac{1}{2} \sum_{i<j} E_{i} E_{j}-\frac{A_{1}^{2}}{2}, \\
& X(z) \equiv \prod_{i}\left(z-E_{i}\right) .
\end{aligned}
$$

This relation may be represented in phase space. In the asymptotic limit, all pairs $\left(b_{n}^{2}, a_{n}\right)$ and $\left(b_{n+1}^{2}, a_{n}\right)$ should lie on a single closed curve (28). The best fit provides the band edges directly. After establishing the $E_{i}$ values, the relation (28) may be used for the extrapolation of coefficients beyond the known values. Fig. 4 shows the plot for the single spinon approximation (which leads to a bounded spectrum) at $k=0$ and $J=0.4$. Several known values and their extrapolation form a single curve. An analogous analysis for different $k$ and $J$ has shown that in the single spinon approximation we have one gap in the continuous part of the spectrum. The asymptotic regime is reached for $n>n_{0} \sim 20$ for $k=0, \pi$. For the more structured spectral density at other $k$ values the asymptotics is reached after $n_{0} \sim 70$. The bounds of the coefficients are

$$
\begin{gathered}
\frac{E_{1}+E_{4}}{2}-g<a_{n}<\frac{E_{1}+E_{4}}{2}+g, g \equiv \frac{E_{3}-E_{2}}{2}, \\
\frac{E_{4}-E_{1}}{4}-\frac{g}{2} \leq b_{n} \leq \frac{E_{4}-E_{1}}{4}+\frac{g}{2}
\end{gathered}
$$

and the half gap value $g \approx 0.074$ equals the amplitude of oscillations. This relation between the amplitude of the coefficient oscillations and the gap does not depend on the total bandwidth $E_{4}-E_{1}$. One may expect that it holds also for an unbounded spectrum. We have modelled such a spectrum by the creation of a gap in the spectral density (23). Then we have calculated the coefficients from the gapped spectral density and we have found that they oscillate around their asymptotics (20) with an amplitude equal to half of the gap. As we have mentioned above, the internal singularities lead to damped oscillations of the coefficients. The restricted number of exact 'bulk-related' ED coefficients makes it impossible at present time to analyse their oscillations in detail and to extract quantitatively the values and positions of the gaps.

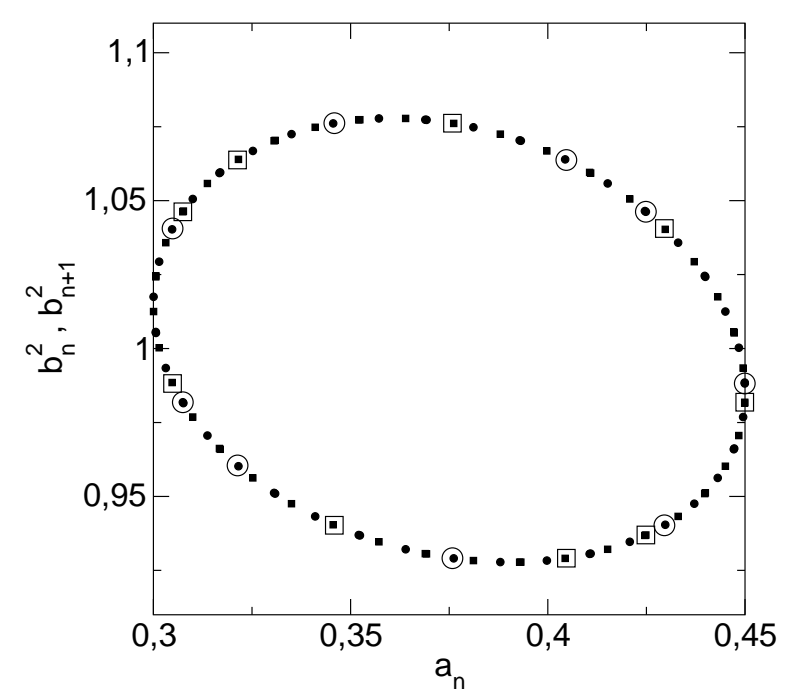

Fig. 4. Phase space representation of the recurrence law (28). The open circles (squares) are pairs of $\left\{b_{n}^{2}, a_{n}\right\}\left(\left\{b_{n+1}^{2}, a_{n}\right\}\right)$, the full symbols correspond to extrapolated values.

\section{Reconstruction of spectral density}

One may summarise the following recipe of spectral density reconstruction from the Lanczos recursion coefficients: i) Establish the number of recursion coefficients that are not strongly affected by the boundaries. ii) Investigate their asymptotics and find a model function that has a continued fraction expansion with similar asymptotics, or equivalently find a reliable procedure to extrapolate the coefficients. iii) Calculate the Green's function $G(k, z)$ using expressions (1926) (then $z$ may be real) or directly (3) (with complex $z$, the numerical efforts depend on the distance to the real axis) up to convergence. Then the spectral density results from the definition (6).

As we have already mentioned, the number of bulkrelated coefficients for the Majumdar-Ghosh model (4) is $n_{0} \approx(L-4) / 2$, where $L$ is the number of sites. We have performed the ED for rings of the length up to 24 sites, i.e. we have about 10 pairs of coefficients for every $k$ value. The last 6 pairs were used for the determination of the parameters of the linear law (20) which are necessary to terminate the continued fraction. Fig. 5 shows the spectral density $A(k, z), z=\omega+\imath \eta$ for $k=\pi / 2, J=0.4$, $\eta=0.01$. On panel (a) the spectral density calculated according to the above recipe (solid line) is compared with the spectral density for the $L=24$ sites ring (dotted line) 

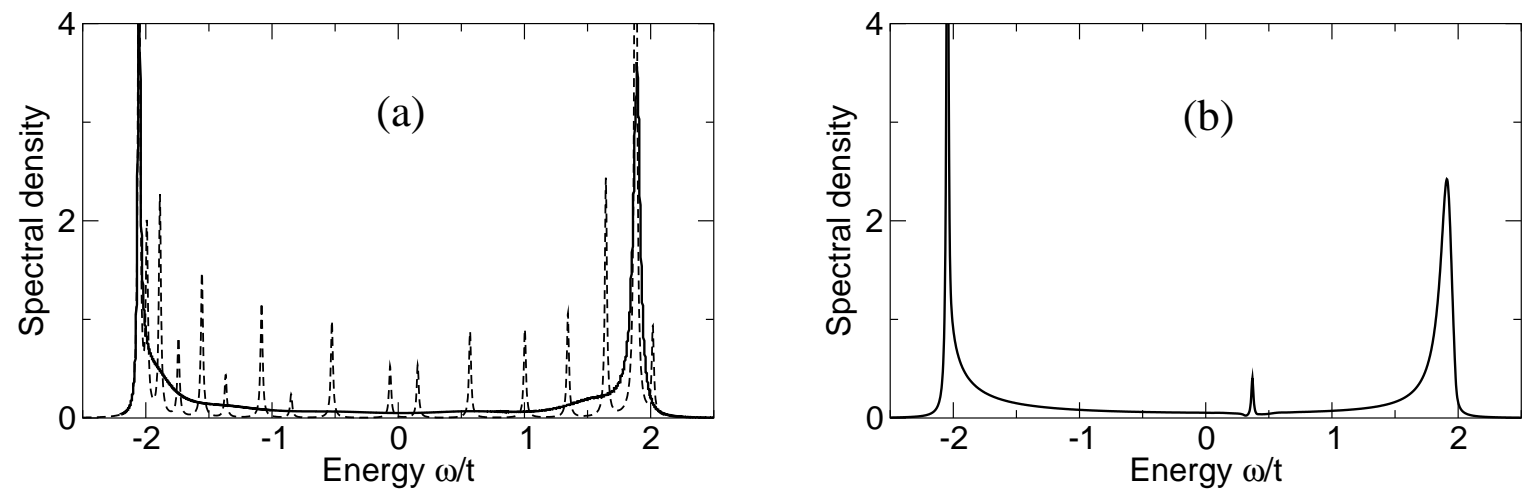

Fig. 5. The spectral density $A(k, \omega+\imath \eta)$ for $k=\pi / 2, J=0.4, \eta=0.01$ (a), compared with the single spinon approximation (b), also for $\eta=0.01$. The solid line in panel (a) corresponds to the analytic termination of the exact Green's function continued fraction expansion after 9 levels. The termination is based on the coincidence of the asymptotic behaviour of the continued fraction expansion of the incomplete Gamma function with the asymptotics of the coefficients shown in Figs. 2 and 3a. The dashed line is the spectral density for the ring with $L=24$ sites.

calculated with the same broadening $\eta$. The qualitative difference of both curves is evident. Panel (b) of the figure shows the result of the single spinon approximation. With a broadening of $\eta=0.01$ the overall shape of the curve is similar to the ED result and is characterised by two peaks at $\omega \approx \pm 2 t$. The small feature near $\omega \sim 0.4$ corresponds to a bound state in the gap, but all bound states and gaps are smeared out for a broadening of $\eta=0.01$ (compare with Fig. $6 \mathrm{~b}$ ). The essential difference with respect to the ED spectrum (for the single spinon approximation the spectrum is bounded) manifests itself as a difference of the relative weight of the peaks, the higher energy peak of ED being smoothed by the exponential tail of the spectral density that tends up to $\omega \rightarrow \infty$.

Fig. 6 shows the spectral density $A(k, \omega+\imath \eta)$ for various $k$-values, $J=0.4$. Panel (a) corresponds to the ED with $\eta=0.01$. The interpretation of the various peaks as different collective excitations were already broadly discussed in Ref. 12: the lower edge of the continuum corresponds to the spinon excitation with a small dispersion of the order $J$. For $k$ different from $\pi / 2$ one can also observe the holon peak with a much larger dispersion (proportional $t$ ) and with a large damping [12].
For the single spinon approximation the law (28) makes it possible to reveal the gap in the continuum part of the spectrum at every $k$ and the existence of quasiparticle states outside the continuum spectrum and within the gap. To show these features a very small broadening of $\eta=0.0001$ was used in panel (b).

\section{Bound states and band gaps}

The existence of a quasiparticle state in the MajumdarGhosh model (i.e. a bound state below the continuum) was proved by ED in Ref. [22] by an analysis of the system size dependence of the pole strength $Z_{h}$ corresponding to the lowest eigenvalue. An analogous way was used to show the existence of a quasiparticle in the single spinon approximation [12] and in the diagrammatic approach [33. It was found that the single spinon approximation gives $Z_{h}$ approximately twice as big as that of the ED. That puzzle can be resolved when one takes into account the linear growth of the recursion coefficients which does not show up in the single spinon approximation. The linear growth may have an influence on the weight $Z_{h}$. From a physical point of view the quasiparticle in this model represents the 

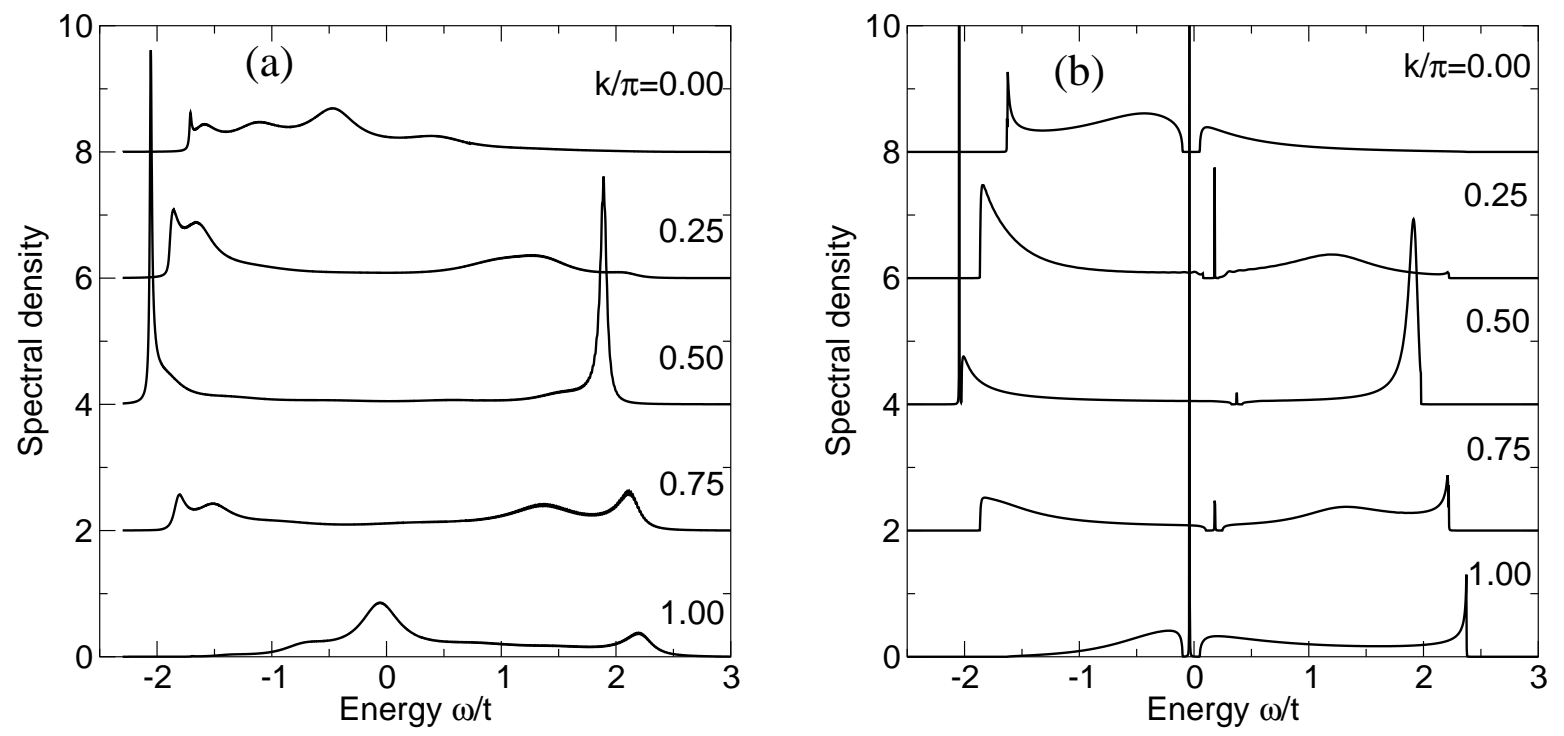

Fig. 6. The spectral density $A(k, \omega+\imath \eta)$ restored from the 'bulk related' coefficients of the exact Green's function continued fraction expansion for various $k$-values, $J=0.4, \eta=0.01$ (a). The spectral density $A(k, \omega+\imath \eta)$ in the single spinon approximation (b) for various $k$-values, $J=0.4$, with $\eta=0.0001$.

bound state of holon and spinon. Scattering processes with the contribution of high energy multispinon excited states that are not taken into account in the single spinon approximation enlarge the probability of decay of the bound state.

Fig. 7 shows the relative weights of the hole wave function decomposition over recursion vectors $\left|u_{n}(k)\right\rangle$ for the three lowest eigenenergies (thick solid, thin solid and dashed lines correspondingly) at the band minimum $k=$ $\pi / 2.90$ recursive coefficients were taken: (a) 'bulk-related' ED coefficients, extrapolated by linear low; (b) single spinon approximation. A qualitatively different behaviour is seen for the spin-polaron quasiparticle wave function (thick line) and the wave functions that belong to the continuum. The different large- $n$ behaviour of recursion coefficients leads to different radius of the polaron state. For comparison, the dotted line on panel (b) shows the change in the wave function caused by the addition of the linear growth (20) to the single spinon approximation coefficients. The linear growth leads to a larger radius of the polaron state and to a decrease of the weight $Z_{h}$ by nearly a factor of two.

The physical origin of the gap in the continuum part of the spectrum that is evident in the single spinon approximation may be understood as follows. Let us recall that in the single spinon approximation we diagonalise the Hamiltonian (4) within the subspace spanned by the operator basis (9). (See the details in Sec. V.B and Appendix C of Ref. [12.) The Green's function (5) for every $k$ is found from the solution of a generalised eigenvalue problem that formally resembles an effective tight-binding model (that is different for every $k$ and is determined by the spin background) with Hamiltonian $E_{r, r^{\prime}}(k)$ in a nonorthogonal basis with overlap matrix $S_{r, r^{\prime}}(k)$. The index $r$ denotes the distance between 'spinon' and 'holon' in (9). The spectral density (6) formally coincides with the density of states of the effective model. In the limit $J, J^{\prime} \rightarrow 0$ both matrices $E$ and $S$ depend only on $r-r^{\prime}$ and the effective model is 'translationally invariant' if we consider $r$ and $r^{\prime}$ as site indices of the effective model. And we obtain the results (1011) making the Fourier transformation over $r-r^{\prime}$. This is the consequence of the degeneracy of all spin states which makes it impossible for the holon to scatter on inhomogeneities of the spin state. By switching on finite $J$ and $J^{\prime}$ the matrix $E_{r, r^{\prime}}(k)$ acquires two contributions. One of them corresponds to the appearance of the spinon coherent motion with the dispersion $\epsilon_{s}(Q)=-2 J \cos Q$. This contribution is also 'translationally invariant' and leads to changes in the spectral density (11) described by (18). Another contribution comes from the commutation of the 'holon end' of the operator (9) with the spin part of the Hamiltonian (4). It describes two 

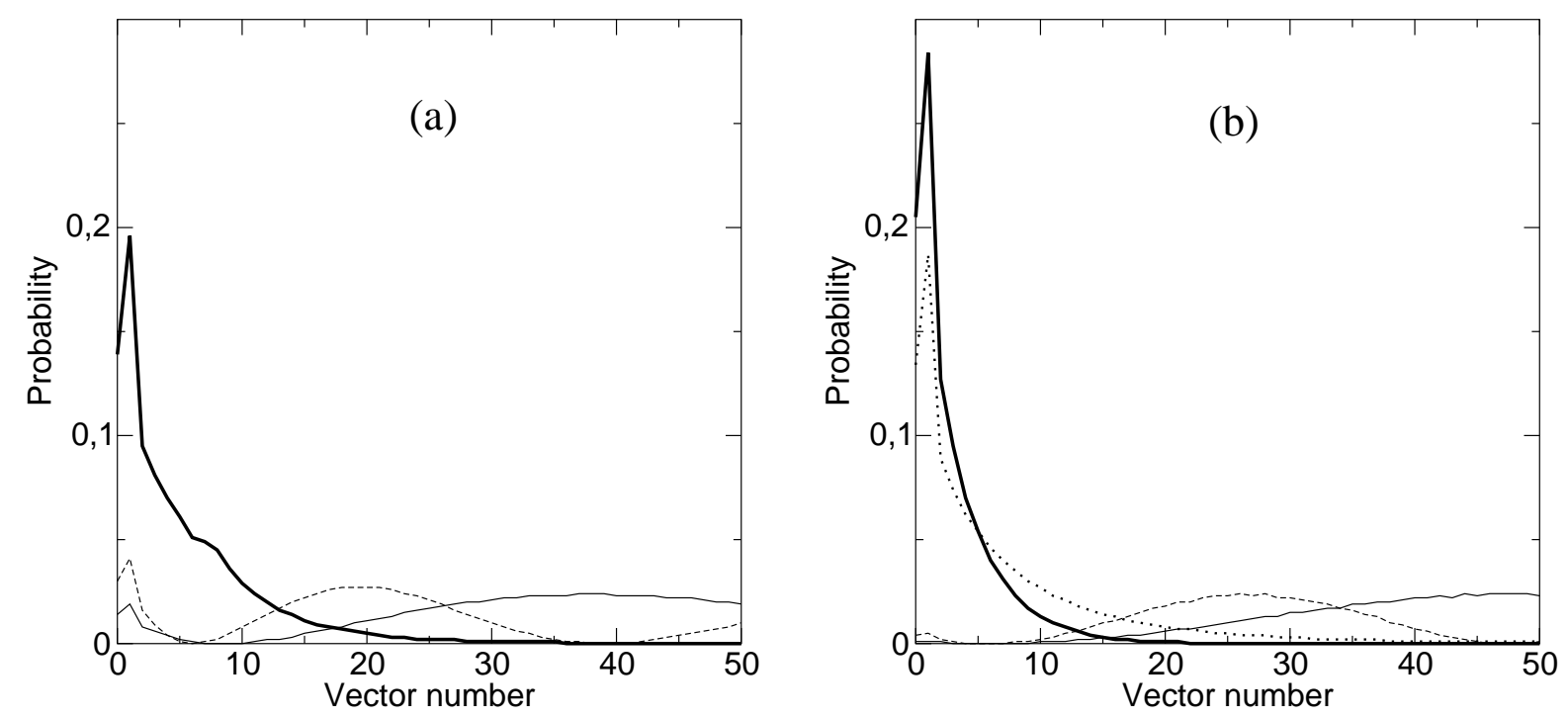

Fig. 7. The relative weights of the wave function decomposition over recursion vectors $\left|u_{n}(k)\right\rangle$ for the three lowest eigenenergies (thick solid, thin solid and dashed lines, correspondingly) at the band minimum $k=\pi / 2$. 90 recursive coefficients were taken: (a) 'bulk-related' ED coefficients, extrapolated by the linear law; (b) single spinon approximation. A qualitatively different behaviour is seen for the spin-polaron quasiparticle wave function (thick line) and the wave functions that belong to the continuum. The different large- $n$ behaviour of the recursion coefficients leads to different radii of the polaron state. For comparison, the dotted line on panel (b) shows the change in the wave function caused by the addition of the linear growth to the coefficients.

processes, the loss of magnetic energy due to the presence of the holon, and the holon-spinon scattering. The former participates in the bound state formation. The latter has a contribution that is different for odd and even $r-r^{\prime}$, i.e. we observe a 'period doubling' in the effective Hamiltonian. This is intimately connected with the dimerised nature of the Majumdar-Ghosh wave function. It is interesting that this feature does not lead to a real period doubling in the system, i.e. the Green's function (5) and the spectral density (6) have the whole Brillouin zone periodicity, as we clearly see in Fig. 6.

\section{Summary}

We propose a new way how to extract the information about the infinite system from the exact diagonalisation of small clusters. It is based on the consideration of Lanczos recursion coefficients that are provided by ED. We have found that the comparison of the results for several cluster sizes allows to obtain the set of the coefficients that are not affected by the finite size effects. These coefficients contain the needed information about the macro- scopic system. When it is possible to infer their asymptotic behaviour (that may strongly differ for the 'bulk related part' compared to the rest of the set for a finite system) we propose to restore the Green's function and spectral density using the terminator technique [8]. This may considerably improve the shape of the spectral density compared to the direct ED result which gives the spectral function in the form of a set of delta functions.

\section{Acknowledgements}

This work was supported by DFG (436 UKR 113/49/41) and by a NATO Collaborative Linkage Grant (PST.CLG. 976416). R.O.K. thanks for hospitality the IFW Dresden, where the main part of this work has been carried out. For several numerical calculations we used the Cambridge Recursion Library 32].

\section{References}

1. P. Fulde, Electron Correlations in Molecules and Solids (Berlin, Springer, 1995). 
2. E. Dagotto, Rev. Mod. Phys. 66, 763 (1994).

3. C. Lanczos, J. Res. Natl. Bur. Stand. 45, 255 (1950).

4. A.C. Hewson, The Kondo Problem to Heavy Fermions (Cambridge University Press, Cambridge, 1997).

5. J. Voit, Rep. Prog. Phys. 57, 977 (1994).

6. R. Haydock, V. Heine, and M.J. Kelly, J. Phys. C: Solid State Phys. 5, 2845 (1972).

7. R. Haydock, in: Solid State Physics 35, eds.: H. Ehrenreich, F. Seitz and D. Turnbull (Academic, New York, 1980).

8. R. Haydock and C.M.M. Nex, J. Phys. C: Solid State Phys. 18, 2235 (1985).

9. P. Turchi, F. Ducastelle, and G. Treglia, J. Phys. C: Solid State Phys. 15, 2891 (1982).

10. V.S. Viswanath and G. Müller, The Recursion Method. Application to Many-Body Dynamics (Berlin, Springer, 1994).

11. C.K. Majumdar and D.K. Ghosh, J. Math. Phys. 10, 1399 (1969); C.K. Majumdar, J. Phys. C 3, 911 (1970).

12. R. Hayn and R.O. Kuzian, Phys. Rev. B 6212156 (2000).

13. E.R. Gagliano and S. Bacci, Phys. Rev. Lett. 621154 (1989).

14. H. Eskes, M.B.J. Meinders, and G.A. Sawatzky, Phys. Rev. Lett. 671035 (1991); W. Stephan and P. Horsch, Phys. Rev. Lett. 66, 2258 (1991); E. Dagotto, A. Moreo, F. Ortolani, J. Riera, and D.J. Scalapino, Phys. Rev. Lett. 67, 1918 (1991); Y. Ohta, K. Tsusui, W. Koshibae, T. Shimotzato, and S. Maekawa, Phys. Rev. B 46, 14022 (1992).

15. C. Kim, A.Y. Matsuura, Z.-X. Shen, N. Motoyama, H. Eisaki, S. Uchida, T. Tohyama, and S. Maekawa, Phys. Rev. Lett. 77, 4054 (1996); K. Kobayashi, T. Mizokawa, A. Fujimori, M. Isobe, and Y. Ueda., Phys. Rev. Lett. 80, 3121 (1998).

16. P.W. Anderson, A Career in theoretical Physics, World Scientific Series in 20th Century Physics (World Scientific, Singapore, 1994), Vol. 7.

17. E.H. Lieb and F.Y. Wu, Phys. Rev. Lett. 20, 1445 (1968).

18. K. Okamoto and K. Nomura, Phys. Lett. A 169, 433 (1992); R. Chitra, S. Pati, H.R. Krishnamurty, D. Sen, and S. Ramasesha, Phys. Rev. B 52, 6581 (1995).

19. S. Sorella and A. Parola, J. Phys. Condens. Matter 4, 3589 (1992); A. Parola and S. Sorella, Phys. Rev. B 45, 13156 (1992).

20. M. Ogata and H. Shiba, Phys. Rev. B 41, 2326 (1990).

21. H. Suzuura and N. Nagaosa, Phys. Rev. B 56, 3548 (1997).

22. T. Tohyama and S. Maekawa, J. Phys. Chem. Solids 59, 1864 (1998).

23. C.M.M. Nex, J. Phys. A: Math. Gen. 11, 653 (1978).

24. A. Magnus, in The Recursion Method and its Applications, eds.: D.G. Pettifor and D.L. Weaire, (Springer, Berlin, 1985).

25. O.A. Starykh and G.F. Reiter, Phys. Rev. B 53, 2517 (1996).

26. Q.F. Zhong and S. Sorella, Phys. Rev. B 51, 16135 (1995).

27 . Strictly speaking, the hole in the $t-J_{z}$ model delocalizes by jumping around an elementary loop 1.5 times 28 . The spectrum considered here corresponds to the retraceable-path approximation.

28. S. Trugman, Phys. Rev. B 37, 1597 (1988).

29. R.O. Kuzian, R. Hayn, A.F. Barabanov, and L.A. Maksimov, Phys. Rev. B 58, 6194 (1998).

30. R.O. Kuzian, R. Hayn, and L.B. Litinskii, Physica B, 259261779 (1999); A.F. Barabanov, R.O. Kuzian, L.A. Maksimov, and E. Zasinas, Zh. Eksp. Theor. Fiz. 113, 1758 (1998) (cond-mat/9702125).
31. W.H. Press, B.P. Flannery, S.A. Teukolsky, and W.T. Vetterling, Numerical Recipes in FORTRAN: The Art of Scientific Computing, (Cambridge University Press, 1986-1992) $\S 6.2$.

32. C.M.M. Nex, Comp. Phys. Comm. 34, 101 (1984); The Cambridge Recursion Library (1986).

33. C. Jurecka and W. Brenig, Phys. Rev. B 63, 094409 (2001). 